\title{
A Banner Year for Global Surgery: Now How to Make it Make a Difference on the Ground
}

\author{
Charles Mock ${ }^{1}$
}

Published online: 16 July 2015

(C) Société Internationale de Chirurgie 2015

People who have been working to assure surgical care to everyone in the world (including that majority of the world's people who live in poverty in low- and middleincome countries (LMICs)) have felt ignored for years by their governments, by international organizations, by the field of global public health and even by many surgical organizations. This has gradually begun to change. This year in particular has seen three notable events/publications related to global surgery: (1) the publication of Disease Control Priorities 3rd Edition (DCP3) Volume 1 Essential Surgery [1]; (2) the publication of the Lancet Commission on Global Surgery [2]; and (3) the adoption of World Health Assembly (WHA) resolution 68.15- "Strengthening emergency and essential surgical care and anaesthesia as a component of universal health coverage" [3]. This editorial will summarize these three events and discuss how the surgical community can put them to use in promoting real, on-the-ground improvements in surgical care globally.

Electronic supplementary material The online version of this article (doi:10.1007/s00268-015-3154-x) contains supplementary material, which is available to authorized users.

Charles Mock-Associate Editor, World Journal of Surgery

Charles Mock

cmock@uw.edu

1 Seattle, WA, USA

\section{Disease Control Priorities}

DCP3 stands as the most comprehensive global effort to evaluate the cost-effectiveness and population-wide effect of health interventions. It has involved the World Bank, the World Health Organization (WHO), the Gates Foundation and several other notable groups. It has been very influential in setting the agenda for many international agencies, donors, and country governments. Prior versions in 1993 and 2006 evaluated several hundred health interventions, including several surgical interventions. The third version (DCP3) is currently underway and is the largest of the three versions, with nine volumes planned. The first of these has now been released: Volume 1. Essential Surgery [1]. This focusses on that component of surgical care that should be the highest priority to implement globally, namely surgical care that addresses conditions that have very large health burdens and for which there are surgical procedures (and related care) that are highly-cost effective and that are feasible to promote globally. The 80 authors and editors of Essential Surgery went on to define a set of 44 procedures (or sets of procedures) that fit these criteria. These procedures primarily cover high burden conditions such as complications of pregnancy, injury, surgical emergencies (e.g. appendicitis), and several congenital anomalies (e.g. cleft). DCP3 estimates that if these procedures could be made available to everyone in the world, 1.5 million deaths could be averted each year. This is eminently feasible to do. These procedures are all very cost-effective, with most costing \$10-\$100 per disability adjusted life year (DALY) averted. This is in the same range as immunizations, bed nets for prevention of malaria, and several other interventions that are considered to be extremely cost-effective, are recommended for wide-scale dissemination, and are the 
focus of multi-billion dollar global programs. The abstract for the first (summary) chapter of the volume is included an appendix in supplementary material to this editorial.

\section{The Lancet Commission on Global Surgery}

The Lancet occasionally has special issues or commissions that study a particular problem and give recommendations. These have been very useful in the past for calling attention to neglected problems and for establishing future directions for specific problems and for health care in general. The Lancet Commission on Global Surgery was begun in 2013 and released in April, 2015. It represented the work of 25 commissioners (from multiple countries and disciplines) along with collaborators from 110 countries. It had five key messages [2];

1. Five billion people do not have access to safe, affordable surgical and anaesthesia care when needed.

2. Of the 313 million surgical procedures carried out each year, only $6 \%$ are done in the world's poorest countries, where a third of the world's people live. 143 million additional surgical procedures are needed in LMICs globally each year.

3. 81 million people face catastrophic expenditures either paying for surgical care or attributable to the nonmedical costs of accessing surgical care.

4. If LMICs were to scale up surgical capacity at the rates achieved by the current best-performing LMICs, most (two-thirds) of countries would be able to reach a minimum operative volume of 5000 surgical procedures per 100,000 population by 2030 .

5. Surgery should be regarded as an "indivisible, indispensable part of health care." [2]

The Commission also developed a set of recommendations to make universal access to safe, affordable surgical care a reality. These include: a set of six indicators that should be tracked and reported by all countries and followed by international organizations, such as the WHO. These include (among others): surgical volume per population, perioperative mortality rate, and surgical workforce density. In research it conducted, the Commission also demonstrated that even a moderate density of 20 specialist surgical, anesthesia, and obstetrical providers per 100,000 population (compared with above 100 in many high-income countries) and a moderate volume of 5000 surgical procedures per 100,000 population (compared with above 10,000 in most high-income countries and even above 20,000 in some) would be sufficient to assure most of the needed surgical procedures.

The Commission also developed a template national surgical plan. This gives recommendations to country governments on how to strengthen delivery of surgical services nationwide through elements of: infrastructure, workforce development, service delivery (e.g. quality improvement programs), financing, and information management. The executive summary of the Lancet Commission report, along with Table 1 (recommended surgical indicators) and Table 7 (template national surgical plan) are included as appendices supplementary material to this editorial.

\section{World Health Assembly resolution on surgical care}

The World Health Assembly (WHA) is the governing board of the WHO. It consists of every minister of health in the world (or his/her designee). It meets periodically at the WHO headquarters in Geneva, Switzerland, to deliberate on global health topics, to set WHO's agenda, and to make suggestions to country governments (WHO Member States). The WHA passes several resolutions each year. These urge Member States to take certain actions related to a particular problem. Resolutions also may request WHO to perform certain actions, such as developing certain guidelines or providing technical assistance to member states. Resolutions are typically developed after lengthy debate, discussion, and negotiation. Resolutions are one of the main mechanisms by which the WHA exercises its oversight of WHO and WHO is held accountable for actions requested in WHA resolutions (with the Director General of the WHO being requested to report back to the WHA in 1-2 years after a resolution on how the WHO has implemented it). Resolutions are usually non-binding on Member States, but still exert considerable influence. For example, a prior resolution on trauma and emergency care (WHA resolution 60.22) led to directly to two major WHO publications on trauma care, one providing technical guidance on quality improvement programs and the other compiling success stories and lessons learned on improving trauma care from multiple countries $[4,5]$. It is more difficult to assess the effect of such resolutions in individual countries. Nonetheless, WHA 60.22 is considered to have been of assistance in influencing several country governments to take concrete action on trauma and emergency care, such as the creation of a national trauma care plan in Thailand (including the designation of a trauma care focal point in the department of health of each province).

This year, for first time ever, the WHA passed a resolution solely on surgical care: WHA resolution 68.15"Strengthening emergency and essential surgical care and anaesthesia as a component of universal health coverage". The broad support for this resolution was evidenced by the unusually high number of countries that sponsored it (nine countries, with representation from four continents). As with other WHA resolutions, it requests actions by both 
Member States and WHO. For example, it requests governments to "carry out regular monitoring and evaluation of the emergency and essential surgical care and anaesthesia capacity of health care facilities to identify unmet...needs...". As another example, it requests WHO to "raise awareness of cost-effective options to reduce morbidity, mortality, and prevent or treat disability and deformity through improved organization and planning of provision of anaesthesia and surgical care that is appropriate for resource constrained countries" [3]. The full text of the WHA resolution can be found at www.who.int.

How to make use of these events and publications to implement real on-the-ground improvements in surgical care

The World Journal of Surgery has been connected with all three endeavors. Both DCP3 and the Lancet Commission generated a considerable amount of research. Much of this research is reported in either the chapters in DCP3 or components/annexes of the Lancet Commission. Some of this research has led to free-standing peer reviewed publications that have been published in WJS. These include several that provide greater detail on the avertable burden estimates developed by DCP3, such as papers on the health burden from surgical conditions in general [6] from congenital anomalies [7] and which give further details on the cost-effectiveness of different aspects of providing care for surgical conditions [8, 9]. These also include papers that give further details on derivation of estimates of the proposed minimum rates of surgery per population from the Lancet Commission [10], recommendations on how surgical services can be strengthened within LMIC health systems [11] and other aspects of the work of the Lancet Commission [12, 13]. Regarding the WHA resolution, an article in this volume gives further details on the origin of the resolution, its specific recommendations, and, importantly, how the surgical community can make use of this resolution [14].

These three events are obviously important milestones. However, they do not lead to improvements in and of themselves. Unless put to use by surgeons and other advocates for surgical care, they risk becoming academic exercises and wasted effort. All three have clear recommendations that need advocacy and political action to make happen. This includes action by multiple stakeholders: such as greater attention to population-wide availability of basic surgical services by the surgical community; development and implementation of national surgical plans by governments; and tracking of indicators of surgical care by international agencies. This editorial in particular calls attention to the article by Price et al. [14] that highlights the steps that the WHA resolution urges country governments to take and that will likely not happen without concerted advocacy by the surgical community. It also highlights steps that the surgical community need to take to disseminate the information and recommendations contained in the resolution.

To help make the recommendations of all three publications reality, WJS calls for papers that represent next steps after these important milestones and provides insights on ways in which their recommendations can be implemented. Multiple avenues can be considered. A few examples include:

- Outcomes of programs and other efforts to increase coverage of the 44 essential procedures recommended by DCP3.

- Implementation of monitoring for the indicators recommended by the Lancet Commission.

- Utilization of capacity assessments (as advocated by the WHA resolution), in particular use of such assessments in the ongoing monitoring of changes over time (instead of just one time surveys) and in response to specific interventions to improve capacity and resultant process and outcome of care at individual hospitals or system-wide.

- All three publications emphasize the need for improvements in quality of care and patient safety. Hence, WJS is particularly interested in reports on outcomes of efforts to improve quality of care, especially if these report on hard outcomes such as changes in process of care and health outcomes (morbidity and mortality).

- All three publications emphasize the potential role of task-sharing in improving access to surgical care in underserved areas. WJS is particularly interested in reports on outcomes of efforts to more widely implement task-sharing, including addressing what is needed to assure its safety, acceptability, and incorporation into nationwide systems of surgical care.

Any articles submitted on these topics should adhere to the World Journal of Surgery policy that data from LMICs should have at least one (preferably more) co-author from that country (preferably as lead author). If it is not possible to have at least one co-author from that country, the reason for this should be explained in the cover letter.

Conflict of interest No benefits in any form have been received or will be received from a commercial party related directly or indirectly to the subject of this article.

\section{References}

1. Debas HT, Donkor P, Gawande A, Jamison DT, Kruk ME, Charles N Mock (Eds) (2015) Disease control priorities: third edition. Volume 1. Essential Surgery.: World Bank. Available from http://www.dcp-3.org/surgery 
2. Meara JG, Leather AJ, Hagander L, Alkire B, Aloson N, Ameh E, et al (2015) Global surgery 2030: evidence and solutions for achieving health, welfare, and economic development: The Lancet Commission on Global Surgery. The Lancet. 2015. Available from http://www.globalsurgery.info/

3. World Health Organization (2015) World Health Assembly resolution 68.15: strengthening emergency and essential surgical care and anaesthesia as a component of universal health coverage. Available from http://apps.who.int/gb/ebwha/pdf_files/WHA68/ A68_R15-en.pdf.Accessed 11 June 2015

4. World Health Organization (2009) Guidelines for trauma quality improvement programmes. World Health Organization, Geneva

5. World Health Organization (2010) Strengthening care for the injured: success stories and lessons learned from around the world. World Health Organization, Geneva

6. Higashi H, Barendregt JJ, Kassebaum NJ, Weiser TG, Bickler SW, Vos T (2015) Burden of injuries avertable by a basic surgical package in low- and middle-income regions: a systematic analysis from the Global Burden of Disease 2010 Study. World J Surg 39:1-9

7. Sitkin N, Ozgediz D, Donkor P, Farmer D (2015) Congenital anomalies in low- and middle-income countries: the unborn child of global surgery. World J Surg 39:36-40

8. Hsia RY, Thind A, Zakariah A, Hicks ER, Mock C (2015) Prehospital and emergency care: updates from the Disease Control Priorities, Version 3. World J Surg. doi:10.1007/s00268-0152997-5
9. Shrime MG, Sleemi A, Ravilla TD (2015) Charitable platforms in global surgery: a systematic review of their effectiveness, costeffectiveness, sustainability, and role training. World J Surg 39:10-20

10. Esquivel M, Molina G, Uribe-Leitz T, Lipsitz S, Rose J, Bickler SW (2015) Proposed minimum rates of surgery to support desirable health outcomes: An observational study based on three strategies. World J Surg. doi:10.1007/s00268-015-3092-7

11. Spiegel DA, Misra M, Bendix P, Hagander L, Bickler SW, Saleh CO (2015) Surgical care and health systems. World J Surg. doi:10.1007/s00268-014-2928-x

12. Chao TE, Patel PB, Kikubaire M, Niescierenko M, Hagander L, Meara JG (2014) Surgical care in Liberia and implications for capacity building. World J Surg. doi:10.1007/s00268-014-2905-4

13. Samad L, Iqbal M, Tariq A, Shahzad W, Khan AJ (2015) Equitable access to comprehensive surgical care: the potential of indigenous private philanthropy in low-income settings. World $\mathbf{J}$ Surg 39:21-28

14. Price R, Makasa E, Hollands M (2015) World Health Assembly resolution 68.15-"Strengthening emergency and essential surgical care and anaesthesia as a component of universal health coverage.": Addressing the public health gaps arising from lack of safe, affordable and accessible surgical and anesthetic services. World J Surg. doi:10.1007/s00268-015-3153-y 